\title{
Theranostics Using Antibodies and Antibody-Related Therapeutics
}

\author{
Kirsten L. Moek*1 ${ }^{1}$, Danique Giesen*1, Iris C. Kok ${ }^{1}$, Derk Jan A. de Groot ${ }^{1}$, Mathilde Jalving ${ }^{1}$, Rudolf S.N. Fehrmann ${ }^{1}$, \\ Marjolijn N. Lub-de Hooge ${ }^{2}$, Adrienne H. Brouwers ${ }^{3}$, and Elisabeth G.E. de Vries ${ }^{1}$ \\ ${ }^{I}$ Department of Medical Oncology, University Medical Center Groningen, University of Groningen, Groningen, The Netherlands; \\ ${ }^{2}$ Department of Clinical Pharmacy and Pharmacology, University Medical Center Groningen, University of Groningen, Groningen, \\ The Netherlands; and ${ }^{3}$ Department of Nuclear Medicine and Molecular Imaging, University Medical Center Groningen, University of \\ Groningen, Groningen, The Netherlands
}

\begin{abstract}
In theranostics, radiolabeled compounds are used to determine a treatment strategy by combining therapeutics and diagnostics in the same agent. Monoclonal antibodies (mAbs) and antibody-related therapeutics represent a rapidly expanding group of cancer medicines. Theranostic approaches using these drugs in oncology are particularly interesting because antibodies are designed against specific targets on the tumor cell membrane and immune cells as well as targets in the tumor microenvironment. In addition, these drugs are relatively easy to radiolabel. Noninvasive molecular imaging techniques, such as SPECT and PET, provide information on the whole-body distribution of radiolabeled mAbs and antibodyrelated therapeutics. Molecular antibody imaging can potentially elucidate drug target expression, tracer uptake in the tumor, tumor saturation, and heterogeneity for these parameters within the tumor. These data can support drug development and may aid in patient stratification and monitoring of the treatment response. Selecting a radionuclide for theranostic purposes generally starts by matching the serum half-life of the mAb or antibody-related therapeutic and the physical half-life of the radionuclide. Furthermore, PET imaging allows better quantification than the SPECT technique. This information has increased interest in theranostics using PET radionuclides with a relatively long physical half-life, such as ${ }^{89} \mathrm{Zr}$. In this review, we provide an overview of ongoing research on mAbs and antibodyrelated theranostics in preclinical and clinical oncologic settings. We identified 24 antibodies or antibody-related therapeutics labeled with PET radionuclides for theranostic purposes in patients. For this approach to become integrated in standard care, further standardization with respect to the procedures involved is required.
\end{abstract}

Key Words: molecular imaging; monoclonal antibodies; oncology; PET; cancer; theranostics

J Nucl Med 2017; 58:83S-90S

DOI: 10.2967/jnumed.116.186940

$\mathbf{T}$ heranostics is a treatment strategy in which a single agent is used for both diagnostic and therapeutic purposes. Theranostic

Received Feb. 2, 2017; revision accepted Apr. 13, 2017.

For correspondence or reprints contact: Elisabeth G.E. de Vries, Department of Medical Oncology, University Medical Center Groningen, University of Groningen, P.O. Box 30.001, 9700 RB Groningen, The Netherlands.

E-mail: e.g.e.de.vries@umcg.nl

${ }^{*}$ Contributed equally to this work.

COPYRIGHT (C 2017 by the Society of Nuclear Medicine and Molecular Imaging. procedures are based on radiolabeling compounds of interest. This approach potentially enables the evaluation of drug target expression and the actual presence of the drug at the tumor site in vivo in cancer patients using imaging methods such as SPECT or PET. Particularly interesting are theranostic approaches using monoclonal antibodies (mAbs) and antibody-related therapeutics because these agents belong to a rapidly expanding group of effective anticancer drugs. Antibody-related therapeutics include bispecific antibodies (e.g., bispecific T-cell engagers [BiTEs]), engineered antibody structures (e.g., minibodies, diabodies, and nanobodies), antibody-drug conjugates (ADCs), and radiolabeled antibodies for radioimmunotherapy. These drugs have ideal characteristics for theranostic approaches because they are designed against a specific target, often on the cell surface, and are relatively easy to radiolabel.

As of December 2016, 24 mAbs or antibody-related therapeutics had been approved by the U.S. Food and Drug Administration (FDA) or the European Medicines Agency (EMA) for use in cancer patients. These drugs comprise 20 mAbs, 1 BiTE, 2 ADCs, and 1 radioimmunotherapy antibody. The approved mAbs and antibody-related therapeutics are directed against targets on the tumor cell membrane and immune cells as well as targets in the microenvironment.

mAbs are administered in noncurative and curative settings. In the noncurative setting, these drugs have proven effects on (diseasefree) survival (1-3). In the adjuvant setting, the anti-human epidermal growth factor receptor 2 (HER2) antibody trastuzumab and the anticytotoxic T-lymphocyte antigen 4 (CTLA-4) antibody ipilimumab increase overall survival in patients with breast cancer and melanoma, respectively $(4,5)$.

In oncology, even when a drug has a proven clinical benefit for a certain patient population, not all patients will benefit. This outcome can potentially be related to heterogeneity in tumor target expression, vascularization of the tumor, or the presence of an immunosuppressive tumor microenvironment. Treatment decisions in both routine practice and drug development are frequently made using information obtained from a biopsy of a single tumor lesion. Furthermore, recommended dosing schedules are mostly determined using blood-based pharmacokinetic analyses. Differences in drug target expression and drug uptake in various tumor lesions within a single patient are almost never considered. In this respect, a theranostic approach is of potential interest because it might provide insight into tumor target heterogeneity and information on whether a drug reaches tumor lesions. For these reasons, molecular antibody imaging can also be a valuable tool in drug development, drug decision making, and patient enrichment strategies. 
In this review, we provide an overview of current research on mAbs and antibody-related therapeutics visualized using PET imaging in both preclinical and clinical oncologic settings.

\section{SEARCH STRATEGY}

To identify available studies investigating theranostic approaches with $\mathrm{mAbs}$ and antibody-related therapeutics, we performed a PubMed search on November 21, 2016. The search terms "PET" AND "Cancer" AND "Antibody" OR "ADC" OR "Bispecific" were used in combination with the most commonly used PET radionuclides: ${ }^{64} \mathrm{Cu},{ }^{68} \mathrm{Ga},{ }^{86} \mathrm{Y},{ }^{89} \mathrm{Zr}$, and ${ }^{124} \mathrm{I}$. We focused on studies published during the last 5 y to capture most recent developments but included relevant studies published earlier. In addition, we searched ClinicialTrials.gov on November 17, 2016, for ongoing studies over the past $10 \mathrm{y}$ with the search terms "Cancer" AND "PET" NOT "FDG." Both searches were limited to articles published in English. Case reports, reviews, and books were excluded. In total, 1,448 preclinical and clinical studies were found. All articles and ongoing studies were manually screened for relevance using the following inclusion criteria. First, a full-sized mAb, ADC, bispecific antibody, or fragment with theranostic potential was used. Second, for a study in which humans were included, the subjects were 18 y old or older. Finally, we limited our search to the most commonly used PET radionuclides to provide a comprehensive overview of relevant agents with prime theranostic potential. Articles were excluded if (potential) theranostic applications of those agents were not found.

\section{GENERAL ASPECTS OF MOLECULAR IMAGING USING MABS AND ANTIBODY-RELATED THERAPEUTICS}

mAbs and antibody-related therapeutics can be efficiently labeled with a wide range of radionuclides. In general, the different labeling techniques can easily be applied to most mAbs and antibody-related therapeutics. These drugs can therefore be used in studies ranging from mice to humans (6).

\section{Chelation and Radiolabeling for Molecular Antibody Imaging}

${ }^{99} \mathrm{~m} \mathrm{Tc},{ }^{64} \mathrm{Cu},{ }^{68} \mathrm{Ga},{ }^{86} \mathrm{Y},{ }^{89} \mathrm{Zr},{ }^{111} \mathrm{In},{ }^{123} \mathrm{I},{ }^{124} \mathrm{I},{ }^{131} \mathrm{I}$, and ${ }^{177} \mathrm{Lu}$ are the radionuclides most commonly used for molecular imaging with mAbs and antibody-related therapeutics in the field of oncology (Table 1). Selecting a suitable radionuclide generally

TABLE 1

Characteristics of Radionuclides Used with mAbs or Antibody-Related Theranostics in Oncology

\begin{tabular}{llrc}
\hline Technique & Isotope & Half-life & Residualizing \\
\hline PET & ${ }^{68 \mathrm{Ga}}$ & $67.7 \mathrm{~min}$ & + \\
& ${ }^{64} \mathrm{Cu}$ & $12.7 \mathrm{~h}$ & + \\
& ${ }^{86 \mathrm{Y}}$ & $14.7 \mathrm{~h}$ & + \\
& ${ }^{89} \mathrm{Zr}$ & $78.4 \mathrm{~h}$ & + \\
& $124 \mathrm{I}$ & $100.3 \mathrm{~h}$ & - \\
& $99 \mathrm{mTc}$ & $6.0 \mathrm{~h}$ & - \\
& ${ }^{123} \mathrm{I}$ & $13.2 \mathrm{~h}$ & + \\
& ${ }^{111} \mathrm{In}$ & $67.3 \mathrm{~h}$ & + \\
& $177 \mathrm{Lu}$ & $159.5 \mathrm{~h}$ & - \\
& $131 \mathrm{I}$ & $192.5 \mathrm{~h}$ & + \\
\hline
\end{tabular}

starts by matching the serum half-life of the mAb or antibodyrelated therapeutic and the physical half-life of the radionuclide. This step is essential to ensure that radioactivity can be detected long enough for the drug to reach its target while minimizing the duration of exposure to harmful radiation (6). The serum half-life mainly depends on the structure and size of the mAb or antibody-related therapeutic. Generally, the serum half-life is shorter for a smaller $\mathrm{mAb}$ construct than for a full-sized $\mathrm{mAb}$ because the molecular weight is often below the renal clearance threshold of approximately $70 \mathrm{kDa}$. For example, the serum halflife of cetuximab $( \pm 150 \mathrm{kDa}$ ) is $3-4 \mathrm{~d}$, whereas the serum halflife of the BiTE antibody blinatumomab $( \pm 60 \mathrm{kDa})$ is only several hours. In addition, the serum half-life depends on the IgG subtype from which the mAb or antibody-related therapeutic is derived and on whether the (constructed) mAb is fully human, humanized murine, or chimeric. The serum half-lives of mAbs and antibodyrelated therapeutics can vary from $30 \mathrm{~min}$ to $30 \mathrm{~d}$.

Furthermore, a chelator is required to link metal-based radionuclides, such as ${ }^{64} \mathrm{Cu},{ }^{68} \mathrm{Ga},{ }^{86} \mathrm{Y},{ }^{89} \mathrm{Zr},{ }^{111} \mathrm{In}$, and ${ }^{177} \mathrm{Lu}$, to an $\mathrm{mAb}$ or antibody-related therapeutic. Deciding on a chelator for human use depends on the radionuclide, the most stable chemical link, and the clinical applicability in terms of validation.

Another important consideration in choosing a nuclide for radiolabeling is whether the $\mathrm{mAb}$ or antibody-related therapeutic becomes internalized after binding to its target. For example, when radiometal-labeled drugs are metabolized, the metal-based radionuclide is trapped intracellularly in lysosomes through residualization (7). This process results in higher absolute uptake of the tracer and leads eventually to higher tumor-to-blood ratios. Iodine-labeled drugs are characterized by rapid renal clearance of the radionuclide from tumor cells because iodinated mAbs do not residualize. However, methods for increasing the internalization of iodine-labeled drugs are available. For instance, a bivalent peptide consisting of $4 \mathrm{D}$-amino acids increased the residence time of the ${ }^{125}$ I radiolabel in renal cell carcinomas (RCCs) significantly over that of the ${ }^{111}$ In-labeled control peptide (8).

\section{Radionuclides Commonly Used in Molecular Antibody Imaging}

Although radionuclides with different physical half-lives are available for radiolabeling, the clinical use of many nuclides is hampered by the requirement for a cyclotron either on-site or about 1 physical half-life of transport time away from the site. An alternative is to use a generator for which a radionuclide laboratory suffices. In the latter situation, the long-lived "mother" radionuclide allows for instant/constant availability of the "daughter" radionuclide. For example, the ${ }^{68} \mathrm{Ga}$ radionuclide is produced using a generator-containing the cyclotronproduced "mother" radionuclide ${ }^{68} \mathrm{Ge}$ - allowing the radiolabeling of mAbs or antibody-related therapeutics at the site of administration. Unfortunately, the relatively short physical half-life (68 $\mathrm{min}$ ) of this radionuclide limits its use for imaging full-sized antibodies, which require several days to achieve sufficient tumor-toblood ratios.

Molecular imaging using the positron emitter ${ }^{89} \mathrm{Zr}$ for antibody labeling has been increasingly used in recent years. This radionuclide has suitable characteristics for molecular antibody imaging; for example, its physical half-life of $78.4 \mathrm{~h}$ generally matches the serum half-life of most mAbs and antibody-related therapeutics in vivo and is compatible with the time needed for residualization, generally allowing high tumor-to-background ratios. Furthermore, 
procedures for the large-scale production of ${ }^{89} \mathrm{Zr}$ have been developed, and mAbs and antibody-related therapeutics can be stably labeled with this radionuclide (9).

\section{Pharmacokinetics and Target Visualization of Radiolabeled mAbs}

Most radiolabeled full-sized antibodies have a relatively long effective half-life (14-21 d). After administration, the drug is distributed throughout the body and taken up by the tumor and other tissues that express its target. Over time, tumor-tobackground ratios will generally increase because of tracer binding to the tumor, residualization of the radiolabel in tumor tissue, and clearance of the nonbound tracer from the circulation and background organs/tissues.

Tumor accumulation of the radiolabeled drug is the consequence of target location, target expression levels, target saturation, and internalization of the drug. In addition, several kinetic features, such as perfusion and vascularization, may influence tumor visualization. For example, tumor uptake of ${ }^{111}$ In-labeled death receptor 5-targeting antibody CS-1008 was observed in only 63\% of 19 patients with metastatic colorectal cancer even though all patients were considered to have death receptor 5-positive lesions (10).

Interestingly, tracer uptake in normal tissues can help explain observed side effects. ${ }^{111}$ In-trastuzumab scintigraphy revealed an increase in myocardial uptake shortly after anthracycline treatment in a subgroup of patients (11). This observation may explain why trastuzumab-related cardiotoxicity can occur when this drug is combined with anthracycline-based chemotherapy.

Clinical imaging studies generally start with determination of the optimal protein dose and time point for exploring tumor-tobackground ratios and image quality (12). Especially for dosedependent pharmacokinetics, the optimal protein dose may have to be high. A radioactive dose of $37 \mathrm{MBq}$ and a scan time of 45$60 \mathrm{~min}$ allow adequate visualization of a ${ }^{89} \mathrm{Zr}$-labeled, full-sized $\mathrm{mAb}$ at days 4-7 $(13,14)$. The $\mathrm{mAb}$ or antibody-related therapeutic is linked to a certain amount of radioactivity per milligramthe so-called specific activity, expressed in $\mathrm{MBq} / \mathrm{mg}$. Specific activities for most $\mathrm{mAbs}$ and antibody-related therapeutics are generally limited to $750-1,000 \mathrm{MBq} / \mathrm{mg}$ because of radiolysis. Unlabeled (naked) antibody is added to the radiolabeled $\mathrm{mAb}$ to allow higher tumor uptake of the tracer for adequate tumor visualization. When the total protein dose that can be safely administered to the patient is relatively low-for example, in the microgram range-reaching a sufficient radioactive dose for successful imaging is difficult. To avoid side effects, the protein dose for T-cell-engaging drugs is generally low, making the use of these drugs as theranostics challenging (15).

\section{USE OF THERANOSTICS IN CLINICAL DECISION MAKING}

We identified 6 different antibody structures that are currently used as theranostic agents in patients. Figure 1 shows how these compounds are directed against a specific target located on the tumor cell or in the tumor microenvironment-for example, macrophages, dendritic cells, and T cells. In addition, Figure 1 shows a simplified illustration of the radiolabeled forms of the therapeutics for theranostic purposes. Most molecular imaging clinical trials have been performed using radiolabeled FDA- or EMA-approved drugs, such as trastuzumab for breast cancer, cetuximab for colorectal cancer, and bevacizumab for several indications (Table 2). An example of ${ }^{89} \mathrm{Zr}$-trastuzumab PET for breast cancer is shown in Figure 2. We identified 14 clinical imaging studies with

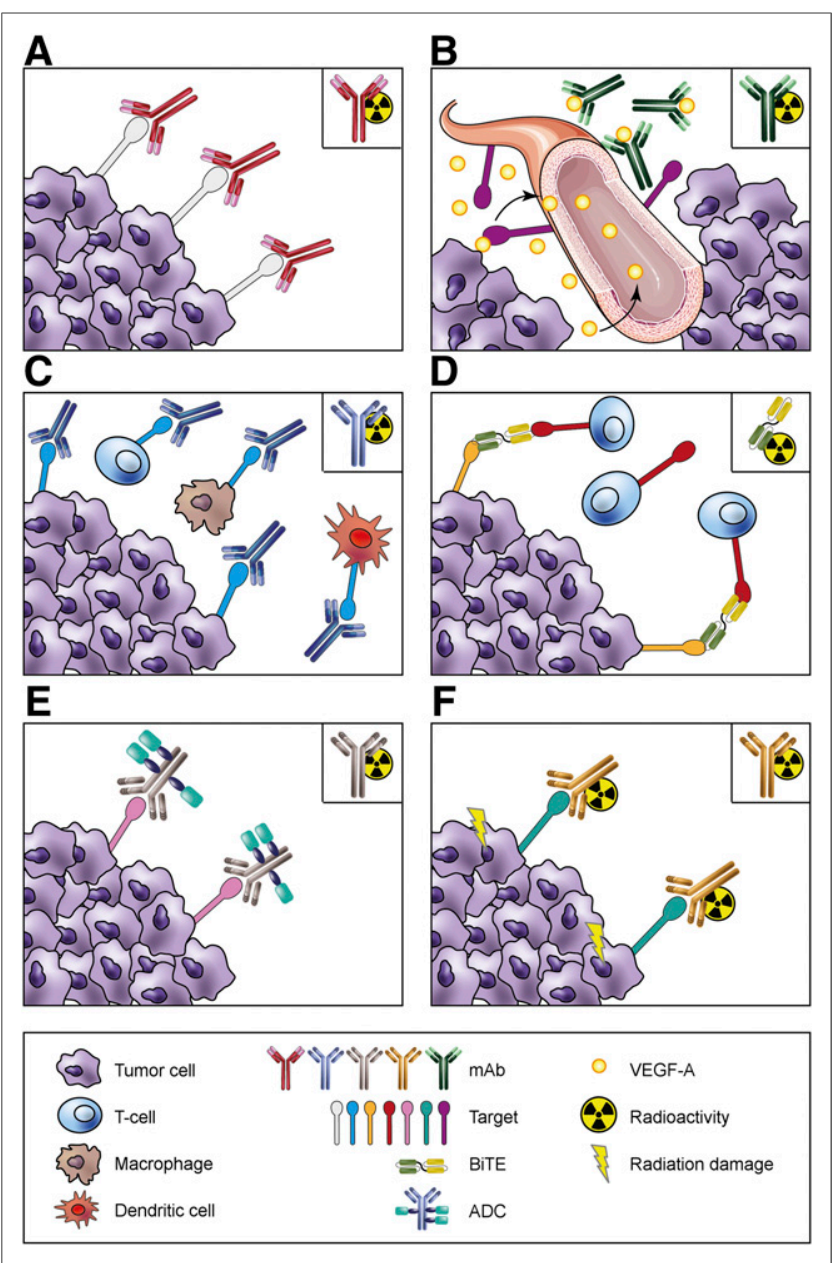

FIGURE 1. Antibodies and antibody-related theranostics. Six different antibody structures in clinical use and radiolabeled compounds used for theranostics (top right corners). (A) Theranostics using mAbs (e.g., trastuzumab targeting HER2 on tumor cells). (B) Theranostics in angiogenesis (e.g., bevacizumab targeting VEGF-A). (C) Theranostics using immune checkpoint inhibitors (e.g., anti-PD-L1 antibody targeting PD-L1 on tumor cells and immune cells). (D) Theranostics using BiTEs (e.g., AMG 211 targeting carcinoembryonic antigen on tumor cells and CD3E on T cells). (E) Theranostics using ADCs (e.g., trastuzumab emtansine targeting HER2 on tumor cells using radiolabeled naked trastuzumab). (F) Theranostics using radioimmunotherapy (e.g., ${ }^{90}$ Y-ibritumomab tiuxetan).

trastuzumab, making this the most frequently investigated therapeutic mAb in molecular imaging (Fig. 1A).

Several lessons can be learned from these studies. First, ${ }^{111} \mathrm{In}-$ trastuzumab SPECT imaging showed new HER2-positive tumor lesions that were not detected using conventional imaging in 13 of 15 metastatic breast cancer patients (16). These data showed that molecular antibody imaging can help identify tumor lesions that are missed by conventional imaging techniques. Second, serial SPECT imaging with ${ }^{111}$ In-trastuzumab before and after 12 wk of trastuzumab treatment showed persistent uptake in all tumor lesions, with only a $20 \%$ decrease in tumor tracer uptake (17). These data indicated that HER 2 is constantly available at the tumor cell surface to bind to trastuzumab and that the tumor is not completely saturated by trastuzumab treatment. Third, in a study with ${ }^{89} \mathrm{Zr}-$ trastuzumab PET for metastatic breast cancer patients, tumor uptake of $10 \mathrm{mg}$ of naked trastuzumab was compared with that of 
TABLE 2

Clinical Studies of Theranostic Uses of Antibodies or Antibody-Related Therapeutics Labeled with PET Radionuclides

\begin{tabular}{|c|c|c|c|c|c|}
\hline Target & Tracer name & Tracer structure & $\begin{array}{c}\text { No. of } \\
\text { (ongoing) } \\
\text { clinical trials }\end{array}$ & Patient population & $\begin{array}{l}\text { No. of } \\
\text { centers }\end{array}$ \\
\hline \multicolumn{6}{|l|}{ Tumor } \\
\hline A33 & ${ }^{124}$ I-huA33 & $\mathrm{mAb}$ & 1 & $\mathrm{CRC}$ & 1 \\
\hline CA6 & ${ }^{64} \mathrm{Cu}-\mathrm{B}-\mathrm{Fab}$ & Fab fragment & 1 & Breast or ovarian cancer & 1 \\
\hline \multirow[t]{2}{*}{ CA9 } & ${ }^{124}$ I-girentuximab & $\mathrm{mAb}$ & 5 & $\mathrm{RCC}$ & 16 \\
\hline & ${ }^{89} \mathrm{Zr}$-girentuximab & $\mathrm{mAb}$ & 2 & $\mathrm{RCC}$ & 3 \\
\hline CEA & 89Zr-AMG 211 & BiTE & 1 & Gastrointestinal adenocarcinoma & 2 \\
\hline CD20 & $\begin{array}{l}{ }^{89} \text { Zr-ibritumomab } \\
\text { tiuxetan }\end{array}$ & $\mathrm{mAb}$ & 1 & $\mathrm{NHL}$ & 1 \\
\hline CD44 & ${ }^{89} \mathrm{Zr}-\mathrm{RG} 7356$ & $\mathrm{mAb}$ & 1 & CD44-positive solid tumor & 6 \\
\hline \multirow[t]{2}{*}{ EGFR (HER1) } & ${ }^{89} \mathrm{Zr}$-cetuximab & $\mathrm{mAb}$ & 4 & CRC, HNSCC, stage IV cancer & 4 \\
\hline & 89Zr-panitumumab & $\mathrm{mAb}$ & 2 & $\begin{array}{l}\text { CRC, NSCLC, sarcoma, urothelial } \\
\text { carcinoma }\end{array}$ & 1 \\
\hline EphA2 & ${ }^{89} \mathrm{Zr}-\mathrm{DS}-8895 \mathrm{a}$ & $\mathrm{mAb}$ & 1 & EphA2-positive cancer & 1 \\
\hline \multirow[t]{4}{*}{ HER2 } & ${ }^{64} \mathrm{Cu}$-trastuzumab & $\mathrm{mAb}$ & 7 & Breast or gastric cancer & 3 \\
\hline & ${ }^{68} \mathrm{Ga}-\mathrm{HER} 2-N a n o b o d y$ & Nanobody & 1 & Breast cancer & 1 \\
\hline & ${ }^{68}$ Ga-trastuzumab-Fab & Fab fragment & 1 & Breast cancer & 1 \\
\hline & ${ }^{89} \mathrm{Zr}$-trastuzumab & $\mathrm{mAb}$ & 7 & Breast cancer & 7 \\
\hline \multirow[t]{3}{*}{ HER3 } & ${ }^{64} \mathrm{Cu}$-patritumab & $\mathrm{mAb}$ & 1 & Solid tumors & 1 \\
\hline & ${ }^{89} Z$ Zr-GSK2849330 & $\mathrm{mAb}$ & 1 & HER3-positive solid tumors & 1 \\
\hline & ${ }^{89} \mathrm{Zr}$-lumretuzumab & $\mathrm{mAb}$ & 1 & HER3-positive solid tumors & 12 \\
\hline MSLN & ${ }^{89} \mathrm{Zr}-\mathrm{MMOT0530A}$ & $\mathrm{mAb}$ & 1 & Ovarian or pancreatic cancer & 2 \\
\hline PIGF & ${ }^{89} \mathrm{Zr}-\mathrm{RO} 5323441$ & $\mathrm{mAb}$ & 1 & GBM & 1 \\
\hline PSCA & ${ }^{124} \mid-A 11$ & Minibody & 1 & $\begin{array}{l}\text { Bladder, pancreatic, or prostate } \\
\text { cancer }\end{array}$ & 1 \\
\hline PSMA & ${ }^{89} \mathrm{Zr}-\mathrm{J} 591$ & $\mathrm{mAb}$ & 4 & GBM, prostate cancer & 2 \\
\hline STEAP1 & ${ }^{89} \mathrm{Zr}-\mathrm{MSTP} 2109 \mathrm{~A}$ & $\mathrm{mAb}$ & 1 & Prostate cancer & 1 \\
\hline \multicolumn{6}{|c|}{ Microenvironment } \\
\hline PD-1 & ${ }^{89} \mathrm{Zr}$-pembrolizumab & $\mathrm{mAb}$ & 1 & NSCLC, melanoma & 2 \\
\hline PD-L1 & ${ }^{89} \mathrm{Zr}$-atezolizumab & $\mathrm{mAb}$ & 1 & Bladder cancer, NSCLC, TNBC & 1 \\
\hline TGF $\beta$ & ${ }^{89} \mathrm{Zr}$-fresolimumab & $\mathrm{mAb}$ & 1 & Glioma & 1 \\
\hline VEGF-A & ${ }^{89} \mathrm{Zr}$-bevacizumab & $\mathrm{mAb}$ & 9 & $\begin{array}{l}\text { Breast cancer, glioma, MM, NET, } \\
\text { NSCLC, RCC }\end{array}$ & 3 \\
\hline
\end{tabular}

$\mathrm{CRC}=$ colorectal carcinoma; CA6 = carbonic anhydrase 6; CA9 = carbonic anhydrase 9; NHL = non-Hodgkin lymphoma; EGFR = epidermal growth factor receptor; HER1 = human epidermal growth factor receptor 1 ; HNSCC $=$ head and neck squamous cell carcinoma; EphA2 = ephrin receptor A2; HER3 = human epidermal growth factor receptor 3; MSLN = mesothelin; PIGF = placental growth factor; GBM = glioblastoma multiforme; PSCA = prostate stem cell antigen; PSMA = prostate-specific membrane antigen; STEAP1 = 6-transmembrane epithelial antigen of prostate family member 1; TNBC = triple-negative breast cancer; TGF $\beta=$ transforming growth factor $\beta$; MM = multiple myeloma; NET = neuroendocrine tumor.

$50 \mathrm{mg}$ of naked trastuzumab, and the tracer dose was also evaluated (13). In trastuzumab-naive patients, $50 \mathrm{mg}$ of naked trastuzumab was needed for adequate imaging, likely because of the dose-dependent pharmacokinetics of trastuzumab. That study showed the relevance of an adequate naked antibody dose for sufficient accumulation of a radiolabeled antibody in the tumor. Fourth, another study showed the value of adding ${ }^{89} \mathrm{Zr}$-trastuzumab PET imaging to biopsies for the assessment of intrapatient tumor heterogeneity and for prediction of the treatment outcome in HER2-positive breast cancer patients treated with trastuzumab emtansine (T-DM1) (18). One third of the patients who had HER2-positive breast cancer showed little or no ${ }^{89} \mathrm{Zr}$-trastuzumab uptake across their metastases and experienced a shorter median time to treatment failure than patients who had more homogeneous HER2-positive PET scan results. These data illustrated a successful theranostic approach for the assessment of tumor heterogeneity and prediction of the treatment outcome. Finally, ${ }^{89} \mathrm{Zr}$-trastuzumab PET imaging can provide a functional readout for therapeutics that affect HER2 expression, 


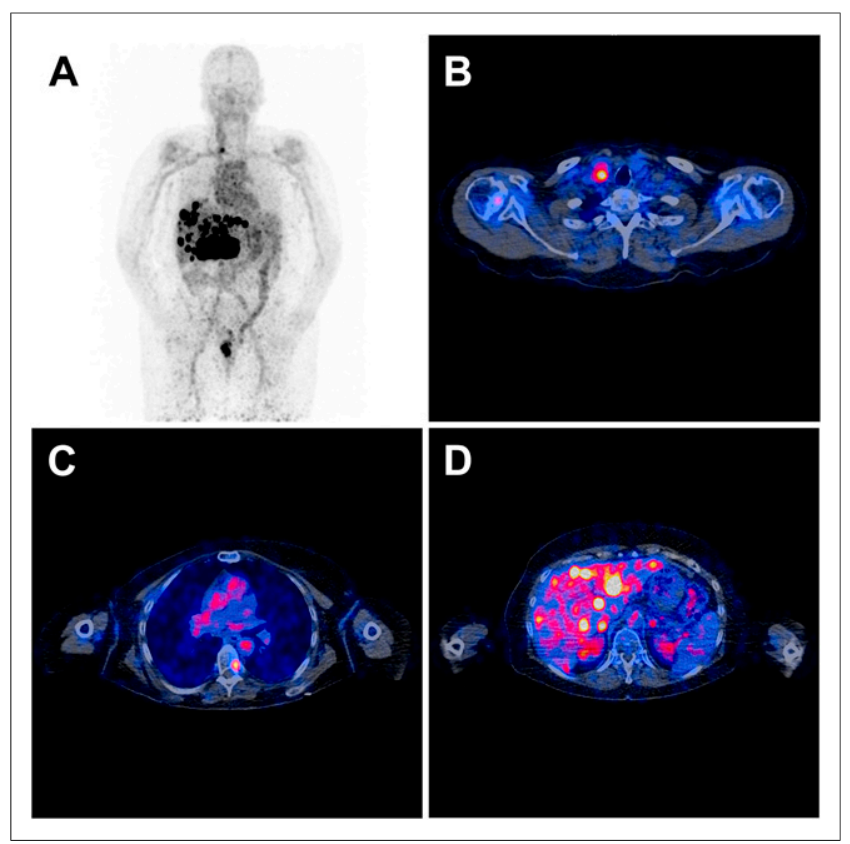

FIGURE 2. ${ }^{89} \mathrm{Zr}$-trastuzumab PET imaging. Patient with human epidermal growth factor receptor 2-positive metastatic breast cancer imaged $4 \mathrm{~d}$ after injection with $37 \mathrm{MBq}$ of ${ }^{89} \mathrm{Zr}$-trastuzumab and total protein dose of $50 \mathrm{mg}$. (A) Maximum-intensity-projection image of ${ }^{89} \mathrm{Zr}$ trastuzumab PET/CT scan showing tracer present in circulation, uptake in intrahepatic metastases, and intestinal excretion. (B) Transverse plane of fused PET/CT (low-dose CT) of chest showing tracer uptake in cervical lymph node. (C) Transverse plane showing tracer uptake in metastasis (left side) in T7. (D) Transverse plane showing tracer uptake in liver metastases.

such as heat shock protein HSP90 inhibitor AUY922 (19). The ongoing IMPACT study is evaluating the clinical utility of ${ }^{89} \mathrm{Zr}$ trastuzumab PET and ${ }^{18} \mathrm{~F}$-fluoroestradiol PET imaging in 200 newly diagnosed metastatic breast cancer patients (ClinicalTrials.gov identifier NCT01957332).

Another well-known drug target is epidermal growth factor receptor 1 , which is targeted by antibodies such as cetuximab and panitumumab. One study demonstrated large differences in tumor ${ }^{89} \mathrm{Zr}$-cetuximab tracer uptake between intrahepatic tumors and extrahepatic tumors in patients with K-ras wild-type metastatic colorectal cancer (14). Extrahepatic tumor uptake of ${ }^{89} \mathrm{Zr}$-cetuximab was demonstrated, whereas liver metastases appeared as "cold spots." Four of 6 patients with ${ }^{89} \mathrm{Zr}$-cetuximab uptake in tumor lesions experienced clinical benefit, whereas progressive disease was observed in 3 of 4 patients without ${ }^{89} \mathrm{Zr}$-cetuximab uptake. Another study with ${ }^{89} \mathrm{Zr}$-cetuximab was performed for head and neck squamous cell cancer (20). In both studies, a therapeutic dose of naked cetuximab followed by ${ }^{89} \mathrm{Zr}$-cetuximab for imaging was used; this protocol may have led to at least partial saturation of the tumor and therefore may have reduced the tumor uptake of ${ }^{89} \mathrm{Zr}$-cetuximab.

Angiogenesis is a hallmark of cancer and is stimulated by vascular endothelial growth factor A (VEGF-A). Several studies have been performed with anti-VEGF-A antibody ${ }^{89} \mathrm{Zr}$-bevacizumab (Fig. 1B) (21-24). They clearly showed that a drug targeting a growth factor in the microenvironment can be visualized using protein tracer doses as low as $5 \mathrm{mg}$. For RCCs, ${ }^{89} \mathrm{Zr}$-bevacizumab PET showed heterogeneous tracer accumulation in tumor lesions
(21). Serial ${ }^{89} \mathrm{Zr}$-bevacizumab PET showed that a therapeutic dose of bevacizumab and interferon- $\alpha$ reduced tracer uptake. This result suggested that 1 therapeutic dose of the angiogenesis inhibitor reduced access of the antibody to the tumor. A ${ }^{89} \mathrm{Zr}$-bevacizumab study of advanced non-small cell lung cancer (NSCLC) demonstrated 4-fold-higher tracer uptake in tumor tissue than in nontumor tissue (22). In children with diffuse intrinsic pontine gliomas treated with radiotherapy, heterogeneity of tumor uptake of ${ }^{89} \mathrm{Zr}$-bevacizumab was shown (23). ${ }^{89} \mathrm{Zr}$-bevacizumab tracer uptake is not limited to malignant disease. In the presence of VEGF-A, benign lesions can also be visualized, as exemplified in patients with von Hippel-Lindau disease (24).

The use of molecular antibody imaging for tumor detection was explored in a large multicenter phase 3 trial in which 14 centers in the United States participated (25). Presurgical ${ }^{124}$ I-girentuximab PET was compared with CT and histopathologic diagnoses in 195 patients with unclassified renal lesions. Girentuximab targets the membrane protein carbonic anhydrase IX, which is expressed in more than $95 \%$ of clear-cell RCCs. ${ }^{124}$ I-girentuximab PET had both better sensitivity and better specificity than $\mathrm{CT}$ for distinguishing clear-cell RCCs from other renal masses, both benign and malignant. That study showed the possibility of performing a novel molecular imaging study across 14 centers. In a multicenter trial of patients who have metastatic RCCs with good or intermediate prognosis, the value of ${ }^{89} \mathrm{Zr}$-girentuximab PET combined with ${ }^{18} \mathrm{~F}$-FDG PET is being tested to determine whether it can help in the selection of patients who have relatively indolent disease-for whom the start of treatment can be postponed (ClinicalTrials.gov identifier NCT02228954).

\section{MOLECULAR IMAGING IN IMMUNOTHERAPY}

Immune checkpoint inhibitors are immunomodulatory mAbs that block immune checkpoints by targeting CTLA-4, programmed death receptor 1 (PD-1), or programmed death receptor 1 ligand (PD-L1) (Fig. 1C). These drugs show activity across multiple tumor types. The immune checkpoint inhibitors ipilimumab (anti-CTLA-4), nivolumab (anti-PD-1), pembrolizumab (anti-PD-1), and atezolizumab (anti-PD-L1) are FDA- and EMA-approved to treat specific tumor types. However, not all patients benefit from these drugs, and patients may experience major immunity-related toxicities. Moreover, these drugs are extremely expensive. Molecular antibody imaging may provide insight into the immune response and may therefore support better patient and treatment selections.

Five preclinical studies with radiolabeled anti-PD-L1 antibodies showed antibody uptake in PD-L1-overexpressing tumors (26-30). These studies provided data on drug biodistribution and the influence of dose escalation on target saturation in mice. In addition to tumor uptake, high tracer uptake was also observed in organs such as the spleen, thymus, and lymph nodes. These data may reflect the expression of PD-L1 by immune cells, including $\mathrm{T}$ cells, dendritic cells, and macrophages.

Three preclinical molecular antibody imaging trials with radiolabeled anti-PD-1 antibodies to visualize $\mathrm{T}$ cells in mice and 1 trial in nonhuman primates have been published (29,31,32). All studies showed tracer uptake patterns to be comparable to those of anti-PD-L1 antibodies in healthy mice, with uptake in tumors and secondary lymphoid organs, such as the spleen and lymph nodes.

The first molecular antibody imaging clinical trials with immune checkpoint inhibitors are under way. One study is investigating the ${ }^{89} \mathrm{Zr}$-labeled anti-PD-L1 antibody atezolizumab 
in patients with bladder cancer, NSCLC, and triple-negative breast cancer (ClinicalTrials.gov identifier NCT02453984), and another is investigating ${ }^{89} \mathrm{Zr}$-labeled anti-PD-1 antibody pembrolizumab in patients with melanoma and NSCLC (ClinicalTrials. gov identifier NCT02760225).

BiTEs represent a relatively novel approach in immunotherapy (Fig. 1D). These bispecific antibodies consist of 2 linked, singlechain variable fragments directed against a surface target antigen on cancer cells and cluster of differentiation $3 \varepsilon$ (CD3E) on $\mathrm{T}$ cells. Simultaneous binding of tumor and $\mathrm{T}$ cells mediated tumor-directed T-cell cytotoxicity and cytokine production without the need for costimulatory molecules (33). Blinatumomab, a CD19/CD3عdirected BiTE, has been approved for the treatment of Philadelphia chromosome-negative relapsed or refractory B-cell precursor acute lymphoblastic leukemia. Two BiTEs have been radiolabeled with ${ }^{89} \mathrm{Zr}$ and studied in mice $(34,35)$. The epithelial cell adhesion molecule-targeting BiTE AMG 110 labeled with ${ }^{89} \mathrm{Zr}$ was used at a $20-\mu \mathrm{g}$ dose in nude BALB/c mice bearing epithelial cell adhesion molecule-expressing colorectal cancer xenografts (34). The highest ${ }^{89} \mathrm{Zr}$-AMG 110 uptake was found in the kidneys and then in the liver and tumor. The CEA/CD3e-directed BiTE AMG 211 radiolabeled with ${ }^{89} \mathrm{Zr}$ showed protein dose-dependent carcinoembryonic antigen (CEA)-specific targeting in mouse tumor xenograft models (35). An ongoing clinical study is exploring the biodistribution of ${ }^{89} \mathrm{Zr}$-AMG 211 in patients with gastrointestinal adenocarcinomas (ClinicalTrials.gov identifier NCT02760199).

\section{USE OF MOLECULAR IMAGING TO STUDY ANTIBODIES WITH PAYLOAD}

ADCs are a subclass of antibody-related therapeutics (Fig. 1E). These drugs consist of a tumor-specific mAb conjugated to a cytotoxic payload via a linker. ADCs are designed to improve the potency of chemotherapy by increasing the accumulation of the cytotoxic drug within neoplastic cells, thereby reducing systemic toxic effects. The antibody part of the ADC does not need to exert a therapeutic effect because it serves as an anchor to deliver cytotoxins directly to cancer cells. Brentuximab vedotin and T-DM1 are the standard of care in, respectively, patients with CD30-positive Hodgkin lymphoma or anaplastic large-cell lymphoma and patients with HER2-overexpressing metastatic breast cancer. More than 80 ADCs are or have been in clinical development.

The only molecular imaging study performed with a radiolabeled ADC involved brentuximab vedotin (36). In mice bearing xenograft tumors with various levels of CD30 expression, tumorto-blood ratios of 15.05 and 0.78 were observed for ${ }^{89} \mathrm{Zr}$-brentuximab vedotin and ${ }^{124}$ I-brentuximab vedotin, respectively, $144 \mathrm{~h}$ after administration. These data suggested that ${ }^{89} \mathrm{Zr}$ was a more suitable radionuclide for this $\mathrm{ADC}$.

Radiolabeling of ADCs themselves is considered to increase the risk of instability of the molecule. Therefore, radiolabeling of the naked antibody that is part of an ADC for PET imaging is a safe alternative. Naked antibody uptake is assumed to reflect ADC uptake and thus may predict whether a patient will respond to ADC therapy. This approach was used in 3 preclinical trials in mice and 1 study in both mice and nonhuman primates (37-40). Organ biodistribution and tracer tumor uptake were assessed. In 1 study, 3 doses of ${ }^{89} \mathrm{Zr}$-labeled naked antibody were evaluated as part of an ADC targeting mesothelin in mice bearing human pancreatic tumor xenografts. Tumor uptake decreased with increasing doses of the naked mAb (38), indicating dose-dependent and saturable tracer distribution at doses of 25 and $100 \mu \mathrm{g}$ in mice. Biodistribution and tumor uptake were also investigated with an ${ }^{89} \mathrm{Zr}$-labeled antimesothelin naked antibody in patients subsequently treated with a mesothelin-directed ADC (41). The results showed uptake of the radiolabeled naked antibody in pancreatic and ovarian tumors. Strickland et al. administered a carcinoembryonic cell adhesion molecule 6-directed ADC to monkeys and assessed biodistribution with a ${ }^{64} \mathrm{Cu}$-labeled anti-carcinoembryonic cell adhesion molecule 6 naked mAb (38). The highest tracer uptake was seen in the bone marrow. Neutropenia and anemia occurred in all animals treated with this ADC, suggesting that tissue-specific toxicity can be predicted by antibody tracer uptake.

In 2 clinical studies, radiolabeled trastuzumab is being evaluated as a biomarker for predicting the response to T-DM1 treatment for HER2-positive metastatic breast cancer. The ZEPHIR trial is designed to prospectively investigate the role of pretreatment ${ }^{89} \mathrm{Zr}$ trastuzumab PET combined with early response assessment using ${ }^{18}$ F-FDG PET in the selection of patients who have metastatic HER2-positive tumors and are unlikely to benefit from T-DM1 treatment (18). An analysis of the data from the first 56 patients showed that negative ${ }^{89} \mathrm{Zr}$-trastuzumab PET findings and the absence of a response in early ${ }^{18} \mathrm{~F}$-FDG $\mathrm{PET}$ resulted in a negative predictive value of $100 \%$ for a response according to RECIST 1.1 criteria. Substantial inter- and intrapatient heterogeneity of tracer uptake was observed. Sixteen of 56 HER2-positive patients (29\%) had negative ${ }^{89} \mathrm{Zr}$-trastuzumab PET results, and intrapatient heterogeneity was detected in $46 \%$ of patients. The same approach is being used to evaluate whether ${ }^{64} \mathrm{Cu}$-labeled trastuzumab can predict a response to T-DM1 therapy (ClinicalTrials.gov identifier NCT02226276).

Antibodies can also function as targeted delivery vehicles for radionuclides as part of radioimmunotherapy to selectively kill tumor cells (Fig. 1F). ${ }^{90}$ Y-ibritumomab tiuxetan has been approved for the treatment of B-cell non-Hodgkin lymphoma. An example of radioimmunotherapy that is being investigated in mice is a ${ }^{177} \mathrm{Lu}-$ labeled anti-CD37 antibody targeting B lymphocytes (42).

\section{TRANSLATION OF MOLECULAR ANTIBODY IMAGING TO CLINICAL PRACTICE}

There are several challenges in translating (pre)clinical antibody imaging studies using theranostics to standardized and, ultimately, daily routine patient care. Knowledge from preclinical models often cannot be extrapolated to humans unconditionally because most antibodies are specific for human targets. In addition, until now, most clinical trials with mAbs or antibody-related therapeutics have been performed in relatively small groups of patients, precluding firm conclusions regarding clinical relevance. Performing larger studies will require harmonization and standardization of radiolabeling and imaging procedures across centers as well as proper access to the required radionuclide. Larger studies using ${ }^{89} \mathrm{Zr}$ are feasible, as the transport of this nuclide or ${ }^{89} \mathrm{Zr}$-labeled drugs can be well organized because of the relatively long physical half-life. The availability of ${ }^{64} \mathrm{Cu}$ is more limited by its relatively fast decay.

When multicenter studies are performed, evidence that the final radiolabeled drug products and manufacturing processes are comparable should be provided for all steps in the manufacturing process that are conducted at more than 1 center. Fortunately, accessing templates for routine documentation, such as Investigational Medicinal Product Dossiers, is becoming easier for $\mathrm{mAbs}$ or antibody-related tracers $(43,44)$. 
We identified 46 medical centers-24 in the United States, 18 in Europe, 3 in Asia, and 1 in Australia-that recently participated in clinical trials with antibodies or antibody-based PET theranostics. ${ }^{89} \mathrm{Zr}$ is by far the most frequently used positron-emitting nuclide for antibody labeling. It is encouraging that of the 24 antibodies or antibody-related therapeutics that have been labeled with several PET radionuclides and investigated as theranostics in patients, 11 were investigated in the multicenter setting.

Finally, the integration of antibody PET imaging in clinical practice is costly. For instance, mAb labeling and a series of PET scans in 1 patient cost several thousand U.S. dollars. However, when proven valuable for making clinical decisions based on whole-body information obtained with molecular antibody imaging, a theranostic approach may prevent expensive treatment of patients who will not benefit from therapy because of a lack of target expression or drug uptake and may therefore lead to fewer side effects and better outcomes.

\section{CONCLUSION}

Theranostics with antibodies and antibody-related therapeutics can provide meaningful in vivo insights about the biodistribution and tumor uptake of radiolabeled drugs. This approach is currently being investigated extensively across numerous centers. Properly powered studies are required to prove that theranostics can play an important role in drug development and become a valuable tool in the selection of patients for antibody-based therapies.

\section{DISCLOSURE}

Elisabeth G.E. de Vries has a consultation/advisory role with Medivation, Merck, and Synthon. Mathilde Jalving has an advisory role with Merck. Research funding was provided by Amgen, Genentech/Roche, Chugai, Servier, Novartis, Synthon, AstraZeneca, and Radius Health. Elisabeth G.E. de Vries received support from an IMPACT grant and RUG 2016-10034 from the Dutch Cancer Society, IMI grant TRISTAN, and ERC advanced grant OnQview. No other potential conflict of interest relevant to this article was reported.

\section{ACKNOWLEDGMENTS}

We thank Anouk Funke and Jan Pruim for their assistance in figure design.

\section{REFERENCES}

1. Loibl S, Gianni L. HER2-positive breast cancer. Lancet. December 6, 2016 [Epub ahead of print].

2. Ribas A, Puzanov I, Dummer R, et al. Pembrolizumab versus investigator-choice chemotherapy for ipilimumab-refractory melanoma (KEYNOTE-002): a randomized, controlled, phase 2 trial. Lancet Oncol. 2015;16:908-918.

3. El-Osta H, Shahid K, Mills GM, Peddi P. Immune checkpoint inhibitors: the new frontier in non-small-cell lung cancer treatment. Onco Targets Ther. 2016;9: 5101-5116.

4. Eggermont AM, Chiarion-Sileni V, Grob JJ, et al. Prolonged survival in stage III melanoma with ipilimumab adjuvant therapy. $N$ Engl J Med. 2016;375: 1845-1855.

5. Perez EA, Romond EH, Suman VJ, et al. Trastuzumab plus adjuvant chemotherapy for human epidermal growth factor receptor 2-positive breast cancer: planned joint analysis of overall survival from NSABP B-31 and NCCTG N9831. J Clin Oncol. 2014;32:3744-3752.

6. Williams SP. Tissue distribution studies of protein therapeutics using molecular probes: molecular imaging. AAPS J. 2012;14:389-399.
7. van Dongen GA, Visser GW, Lub-de Hooge MN, de Vries EG, Perk LR. Immuno-PET: a navigator in monoclonal antibody development and applications. Oncologist. 2007;12:1379-1389.

8. van Schaijk FG, Broekema M, Oosterwijk E, et al. Residualizing iodine markedly improved tumor targeting using bispecific antibody-based pretargeting. J Nucl Med. 2005;46:1016-1022.

9. Verel I, Visser GW, Boellaard R, Stigter-van Walsum M, Snow GB, van Dongen GA. ${ }^{89} \mathrm{Zr}$ immuno-PET: comprehensive procedures for the production of ${ }^{89} \mathrm{Zr}$-labeled monoclonal antibodies. J Nucl Med. 2003;44:1271-1281.

10. Ciprotti M, Tebbutt NC, Lee FT, et al. Phase 1 imaging and pharmacodynamic trial of CS-1008 in patients with metastatic colorectal cancer. J Clin Oncol. 2015;33:2609-2616.

11. de Korte MA, de Vries EG, Lub-de Hooge MN, et al. ${ }^{111}$ Indium-trastuzumab visualises myocardial human epidermal growth factor receptor 2 expression shortly after anthracycline treatment but not during heart failure: a clue to uncover the mechanisms of trastuzumab-related cardiotoxicity. Eur J Cancer. 2007;43:2046-2051.

12. Lamberts LE, Williams SP, Terwisscha van Scheltinga AGT, et al. Antibody positron emission tomography imaging in anticancer drug development. J Clin Oncol. 2015;33:1491-1504.

13. Dijkers EC, Oude Munnink TH, Kosterink JG, et al. Biodistribution of ${ }^{89} \mathrm{Zr}$ trastuzumab and PET imaging of HER2-positive lesions in patients with metastatic breast cancer. Clin Pharmacol Ther. 2010;87:586-592.

14. Menke-van der Houven van Oordt CW, Gootjes EC, Huisman MC, et al. ${ }^{89} \mathrm{Zr}-$ cetuximab PET imaging in patients with advanced colorectal cancer. Oncotarget. 2015;6:30384-30393.

15. Tibben JG, Boerman OC, Massuger LF, Schijf CP, Claessens RA, Corstens FH. Pharmacokinetics, biodistribution and biological effects of intravenously administered bispecific monoclonal antibody OC/TR $\mathrm{F}\left(\mathrm{ab}^{\prime}\right) 2$ in ovarian carcinoma patients. Int J Cancer. 1996;66:477-483.

16. Perik PJ, Lub-De Hooge MN, Gietema JA, et al. Indium-111-labeled trastuzumab scintigraphy in patients with human epidermal growth factor receptor 2positive metastatic breast cancer. J Clin Oncol. 2006;24:2276-2282.

17. Gaykema SBM, de Jong JR, Perik PJ, et al. ${ }^{111}$ In-trastuzumab scintigraphy in HER2-positive metastatic breast cancer patients remains feasible during trastuzumab treatment. Mol Imaging. 2014;13:1-6.

18. Gebhart G, Lamberts LE, Wimana Z, et al. Molecular imaging as a tool to investigate heterogeneity of advanced HER2-positive breast cancer and to predict patient outcome under trastuzumab emtansine (T-DM1): the ZEPHIR trial. Ann Oncol. 2016;27:619-624.

19. Gaykema SBM, Schroder CP, Vitfell-Rasmussen J, et al. ${ }^{89} \mathrm{Zr}$-trastuzumab and ${ }^{89} \mathrm{Zr}$ bevacizumab PET to evaluate the effect of the HSP90 inhibitor NVP-AUY922 in metastatic breast cancer patients. Clin Cancer Res. 2014;20:3945-3954.

20. Even AJ, Hamming-Vrieze O, van Elmpt W, et al. Quantitative assessment of zirconium-89 labeled cetuximab using PET/CT imaging in patients with advanced head and neck cancer: a theragnostic approach. Oncotarget. 2017;8: 3870-3880.

21. Oosting SF, Brouwers AH, van Es SC, et al. ${ }^{89} \mathrm{Zr}$-bevacizumab PET visualizes heterogeneous tracer accumulation in tumor lesions of renal cell carcinoma patients and differential effects of antiangiogenic treatment. J Nucl Med. 2015;56:63-69.

22. Bahce I, Huisman MC, Verwer EE, et al. Pilot study of ${ }^{89} \mathrm{Zr}$-bevacizumab positron emission tomography in patients with advanced non-small cell lung cancer. EJNMMI Res. 2014;4:35.

23. Jansen MH, Veldhuijzen van Zanten SEM, van Vuurden DG, et al. Molecular drug imaging: ${ }^{89} \mathrm{Zr}$-bevacizumab PET in children with diffuse intrinsic pontine glioma. J Nucl Med. 2017;58:711-716.

24. Oosting SF, van Asselt SJ, Brouwers AH, et al. ${ }^{89} \mathrm{Zr}$-bevacizumab PET visualizes disease manifestations in patients with von Hippel-Lindau disease. J Nucl Med. 2016;57:1244-1250.

25. Divgi CR, Uzzo RG, Gatsonis C, et al. Positron emission tomography/computed tomography identification of clear cell renal cell carcinoma: results from the REDECT trial. J Clin Oncol. 2013;31:187-194.

26. Heskamp S, Hobo W, Molkenboer-Kuenen JD, et al. Noninvasive imaging of tumor PD-L1 expression using radiolabeled anti-PD-L1 antibodies. Cancer Res. 2015;75:2928-2936.

27. Chatterjee S, Lesniak WG, Gabrielson M, et al. A humanized antibody for imaging immune checkpoint ligand PD-L1 expression in tumors. Oncotarget. 2016;7:10215-10227.

28. Lesniak WG, Chatterjee S, Gabrielson M, et al. PD-L1 detection in tumors using $\left[{ }^{64} \mathrm{Cu}\right]$ atezolizumab with PET. Bioconjug Chem. 2016;27:2103-2110.

29. Hettich M, Braun F, Bartholoma MD, Schirmbeck R, Niedermann G. Highresolution PET imaging with therapeutic antibody-based PD-1/PD-L1 checkpoint tracers. Theranostics. 2016;6:1629-1640. 
30. Josefsson A, Nedrow JR, Park S, et al. Imaging, biodistribution, and dosimetry of radionuclide-labeled PD-L1 antibody in an immunocompetent mouse model of breast cancer. Cancer Res. 2016;76:472-479.

31. Cole E, Kim J, Donnelly D, et al. Radiosynthesis and preclinical PET evaluation in healthy non-human primates of ${ }^{89} \mathrm{Zr}$-nivolumab [abstract]. $\mathrm{J}$ Nucl Med. 2016;57(suppl 2):1189.

32. England CG, Ehlerding EB, Hernandez R, et al. Preclinical pharmacokinetics and biodistribution studies of ${ }^{89} \mathrm{Zr}$-labeled pembrolizumab. J Nucl Med. 2017;58:162-168.

33. Brischwein K, Parr L, Pflanz S, et al. Strictly target cell-dependent activation of $\mathrm{T}$ cells by bispecific single-chain antibody constructs of the BiTE class. $J$ Immunother. 2007;30:798-807.

34. Warnders FJ, Waaijer SJ, Pool M, et al. Biodistribution and PET imaging of labeled bispecific T cell-engaging antibody targeting EpCAM. J Nucl Med. 2016;57:812-817.

35. Waaijer SJH, Warnders FJ, Lub-de Hooge MN, et al. Preclinical evaluation of the radiolabeled bispecific T-cell engager ${ }^{89} \mathrm{Zr}$-AMG 211 targeting CEA-positive tumors [abstract]. Mol Cancer Ther. 2015;14(12 suppl 2):A85.

36. Moss A, Gudan J, Albertson T, Whiting N, Law C. Preclinical microPET/CT imaging of ${ }^{89} \mathrm{Zr}$-Df-SGN-35 in mice bearing xenografted CD30 expressing and non-expressing tumors [abstract]. Cancer Res. 2014;74(19 suppl):104.

37. ter Weele EJ, Terwisscha van Scheltinga AGT, Kosterink JGK, et al. Imaging the distribution of an antibody-drug conjugate constituent targeting mesothelin with
${ }^{89} \mathrm{Zr}$ and IRDye $800 \mathrm{CW}$ in mice bearing human pancreatic tumor xenografts. Oncotarget. 2015;6:42081-42090.

38. Strickland LA, Ross J, Williams S, et al. Preclinical evaluation of carcinoembryonic cell adhesion molecule (CEACAM) 6 as potential therapy target for pancreatic adenocarcinoma. J Pathol. 2009;218:380-390.

39. Ilovich O, Natarajan A, Hori S, et al. Development and validation of an immunoPET tracer as a companion diagnostic agent for antibody-drug conjugate therapy to target the CA6 epitope. Radiology. 2015;276:191-198.

40. Rylova SN, Del Pozzo L, Klingeberg C, et al. Immuno-PET imaging of CD30positive lymphoma using ${ }^{89} \mathrm{Zr}$-desferrioxamine-labeled CD30-specific AC-10 antibody. J Nucl Med. 2016;57:96-102.

41. Lamberts LE, Menke-van der Houven van Oordt CW, ter Weele EJ, et al. ImmunoPET with anti-mesothelin antibody in patients with pancreatic and ovarian cancer before anti-mesothelin antibody-drug conjugate treatment. Clin Cancer Res. 2016;22:1642-1652.

42. Repetto-Llamazares AH, Larsen RH, Giusti AM, et al. ${ }^{177}$ Lu-DOTA-HH1, a novel anti-CD37 radio-immunoconjugate: a study of toxicity in nude mice. PLoS One. 2014;9:e103070.

43. IND regulatory \& manufacturing resources. National Cancer Institute website. https://imaging.cancer.gov/programs_resources/IND_regulatory_manufacturing. htm. Updated October 28, 2016. Accessed May 15, 2017.

44. Todde S, Windhorst AD, Behe M, et al. EANM guideline for the preparation of an Investigational Medicinal Product Dossier (IMPD). Eur J Nucl Med Mol Imaging. 2014;41:2175-2185. 